\title{
Measurement and testing of lidarsensors
}

\author{
Andy Günther, B. Bäker
}

IAD, TU Dresden

This manuscript is not available according to publishing restriction.

Thank you for your understanding. 